\title{
Fabrication of Micropits by LIBWE for Laser Marking of Glass Materials
}

\author{
Tadatake Sato*1, Aiko Narazaki ${ }^{* 1,}$ and Hiroyuki Niino*1 \\ ${ }^{* 1}$ Research Institute for Sustainable Chemistry, National Institute of Advanced Industrial Science \\ and Technology (AIST), Tsukuba Central 5, 1-1-1 Higashi, Tsukuba, Ibaraki 305-8565, Japan \\ E-mail: sato-tadatake@aist.go.jp
}

\begin{abstract}
Fabrication of micropit array structures on silica glass by fast beam-scanning LIBWE was employed for laser marking of glass materials. Microstructures were fabricated by LIBWE employing a DPSS laser ( $\lambda=355 \mathrm{~nm}, \tau \sim 15 \mathrm{~ns}$ ) and toluene saturated with pyrene. When the beam scanning speed is low, microtrenches with $30 \mu \mathrm{m}$ width are formed. On the other hand, micropit array structures can be fabricated under fast beam scanning conditions. Each micropit in an array structure obtained by single scan was fabricated by single laser pulse irradiation. Micropits with maximum depths of up to $0.35 \mu \mathrm{m}$ could be fabricated by single laser pulse irradiation. For fabricating micropit array structures, the pulse repetition rate becomes an important parameter, since cavitation limits the etching at high pulse repetition rates. The effect of cavitation on etching was investigated by the measurement of transient pressure signals observed upon single and double pulse irradiation. Fabricated micropit array structures can be utilized for fast laser marking of glass materials.
\end{abstract}

DOI: 10.2961/jlmn.2017.03.0013

Keywords: laser direct write, laser-micromachining, laser marking, LIBWE, cavitation

\section{Introduction}

Variable information technologies, that can be attained by laser marking, have been widely used for product identification, counterfeit protection, tracking production and so on [1-5]. This situation would be owing to the advantages of laser marking: flexibility and speed. Mask-less marking can be done by direct writing with computer-controlled optical scanning [6]. The combination of a galvano-scanner and a diode-pumped solid state (DPSS) laser with a high pulse repetition up to few tens $\mathrm{kHz}$ can contribute to high speed flexible marking. Currently, many laser marker systems of this combination are commercially available.

Laser marking can be applied to various materials such as plastics [1, 6, 7], ceramics [1, 6], and metals [2, 4, 5, 7]. For glass materials, a data matrix was written by using laser-induced damages [8]. Such damages often result in deterioration in the strength of the glassware, since they could trigger crack formation.

Crack-free surface micromachining of glass materials could be done by mean of a laser-induced backside wet etching (LIBWE) [9-31], that is an indirect laser processing technique employing organic dye solution as laserabsorbing media. Initially, LIBWE is done by employing an excimer laser and mask-projection systems [9, 16, 22]. While the combination of a DPSS laser and a galvanoscanner was applied for LIBWE as well [12]; direct writing of a LOGO mark composed of aligned microtrenches was reported. Microtrenches are fabricated by overlapping of irradiated laser pulses at high pulse repetition rates. When scan speed increased enough for discrete irradiation, etching by each laser pulses becomes discernable and can be used for high-speed and flexible marking [13, 15]. Ultimately, usage of microstructures fabricated by each laser pulse would contribute to the fastest drawing of various marks or information. Thus, micropits fabricated by laser pulses in LIBWE could be used for high-speed laser marking of glassware. Meanwhile, it was reported that LIBWE process involved the generation of cavitation bubbles [10, $13,14,18,23,28,30]$. In our previous report, the decrease in etch rate was shown and discussed on the basis of temporal loss of the glass-liquid interface [14].

In this work, fabrication of micropits array structures by fast beam scanning was examined for laser marking of glass materials. Etching behavior was investigated with relevance to the cavitation behaviors.

\section{Experimental}

For the LIBWE processes, a combination of a galvanometer-based optical scanning system and a diodepumped solid state (DPSS) laser with a high pulse repetition was employed (Fig. 1). Third-harmonic-generated (THG) pulses of a single-mode DPSS laser, a Nd:YVO laser (Coherent, AVIA355-4500, $\lambda=355 \mathrm{~nm}, \tau \sim 15 \mathrm{~ns}, \mathrm{M}^{2}$ $<1.3$ ), were used for irradiation. The laser was operated at the repetition rate of $5-40 \mathrm{kHz}$.

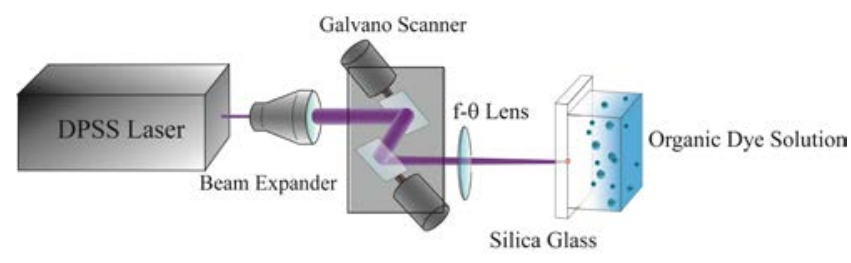

Fig. 1 An experimental setup for beam-scanning LIBWE. 
The laser beam with a nominal diameter of $3.5 \mathrm{~mm}$ was expanded 2 times with a zoom-beam-expander (Sill Optics), and scanned with galvano-scanner (YE Data Inc., MIRAMOTION). Scanned beam was focused by means of a $\mathrm{f}-\theta$ lens (Sill Optics, S4LFT5259, $\mathrm{f}=256.82 \mathrm{~mm}$ ). The beam diameter at the focal point was calculated to be 22 $\mu \mathrm{m}$.

A fused-silica glass plate (Tosoh SGM Co., ES grade) with a thickness of $2 \mathrm{~mm}$ was used as a sample. Pyrene dissolved in toluene with saturated concentration was used as an organic dye solution for LIBWE.

Cavitation behaviors were monitored by measuring transient pressure signals with a piezoelectrical PVDF transducer (Dr. Müller Ingenieurtechnik, Müller-PlatteGauge, Germany, M60-1L with Coating, rise time: $90 \mathrm{~ns}$ ) $[11,13]$. A gap between glass surface and sensor was set to be $2.0 \mathrm{~mm}$. The signals were recorded by a digital oscilloscope that allows fast digitizing of long-duration events (LeCroy WaveJet 354A).

Fabricated microstructures were observed by means of a laser confocal microscope (Keyence, VK-8500). Depthprofiles of the surface were measured by the laser confocal microscope.

\section{Results and Discussions}

\subsection{LIBWE with fast scanning}

There are several reports on LIBWE employing DPSS laser with high pulse repetition [10,13-15]. In these works, laser pulses were scanned by means of a galvano-scanner. When laser beam with a pulse energy of $50.6 \mu \mathrm{J} / \mathrm{pulse}$ and a pulse repetition of $10 \mathrm{kHz}$ was scanned with the speed of $60 \mathrm{~mm} / \mathrm{s}$, a microtrench was formed with single scan as shown in Fig. 2.

(a)

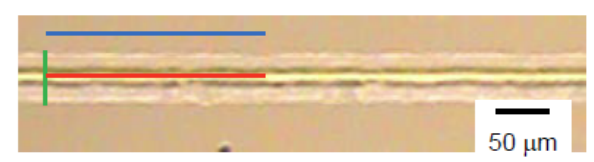

(b)

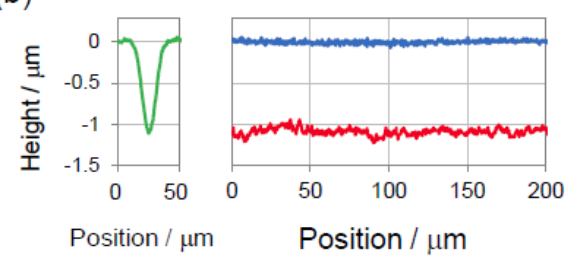

Fig. 2 (a) A micrograph of the microstructure fabricated with beam scanning (pulse energy: $50.6 \mu \mathrm{J} /$ pulse, pulse repetition: $10 \mathrm{kHz}$, scan speed $60 \mathrm{~mm} / \mathrm{s}$ ) and

(b) surface profiles obtained along the colored lines shown in the micrograph.

Under the conditions, distance between two successive irradiated spots was $6 \mu \mathrm{m}$. The trench showed almost homogeneous depth. Meanwhile, a cross-sectional shape reflecting the Gaussian beam profile was observed (Fig. 2(b)). The width of fabricated microtrench was ca. $30 \mu \mathrm{m}$, which reveals a diameter of the irradiated beam spots effective for the etching. This effective beam diameter was used for calculating applied fluence as an averaged value for whole Gaussian beam area: in this case, fluence was estimated to be $7.2 \mathrm{~J} / \mathrm{cm}^{2}$. In many works on LIBWE employing homogenized laser beam, etching behavior generally discussed on the basis of laser fluence. Calculated fluence for focused Gaussian beam is generally high compared with those employed in LIBWE with homogenized laser beam $\left(\sim 2 \mathrm{~J} / \mathrm{cm}^{2}\right)$.

When scan speed was increased to $300 \mathrm{~mm} / \mathrm{s}$, distance between two successive irradiated spots was $30 \mu \mathrm{m}$, a fabricated microstructure changed into an array of micropits with diameters of ca. $30 \mu \mathrm{m}$, as shown in Fig. 3. The observed microstructure indicates that these micropits were fabricated by pulse to pulse in one scan. Thus, the maximum depths of each micropit of $0.35 \mu \mathrm{m}$ was obtained under single pulse irradiation.

(a)

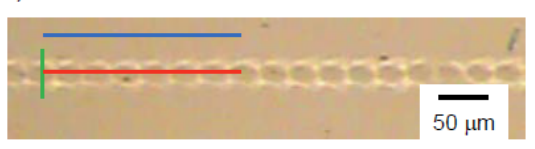

(b)

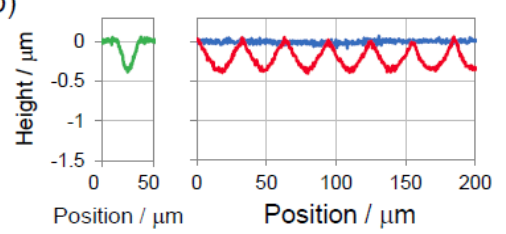

Fig. 3 (a) A micrograph of the microstructure fabricated with beam scanning (pulse energy: $50.6 \mu \mathrm{J} / \mathrm{pulse}$, pulse repetition: $10 \mathrm{kHz}$, scan speed $300 \mathrm{~mm} / \mathrm{s}$ ) and (b) surface profiles obtained along the colored lines shown in the micrograph.

\subsection{Micropit arrays formed by LIBWE}

Micropit arrays fabricated with LIBWE were investigated in detail. Maximum depths of micropits fabricated with beam scanning at $300 \mathrm{~mm} / \mathrm{s}$ increased linearly with pulse energies as shown in Fig. 4. Micropits with the maximum depth of up to $0.35 \mu \mathrm{m}$ could be fabricated by single pulse irradiation without crack formation. Linear increase was observed for the results obtained with pulse energies higher than $23.1 \mu \mathrm{J} / \mathrm{pulse}$. The fitted line showed the Xintercept at $14.6 \mu \mathrm{J} /$ pulse. Whereas, obtained maximum depths were suddenly dropped to zero at the pulse energy of $19.9 \mu \mathrm{J} /$ pulse. This pulse energy corresponds to fluence of $2.8 \mathrm{~J} / \mathrm{cm}^{2}$. Although resemble behavior in etch rates was reported by Bohme and Zimmer [17], results shown in Fig. 4 are not etch rates but depths of structure obtained by single pulse irradiation. Sudden change observed at 19.9 $\mu \mathrm{J} /$ pulse would be due to incubation effect becoming apparent as discussed later. 


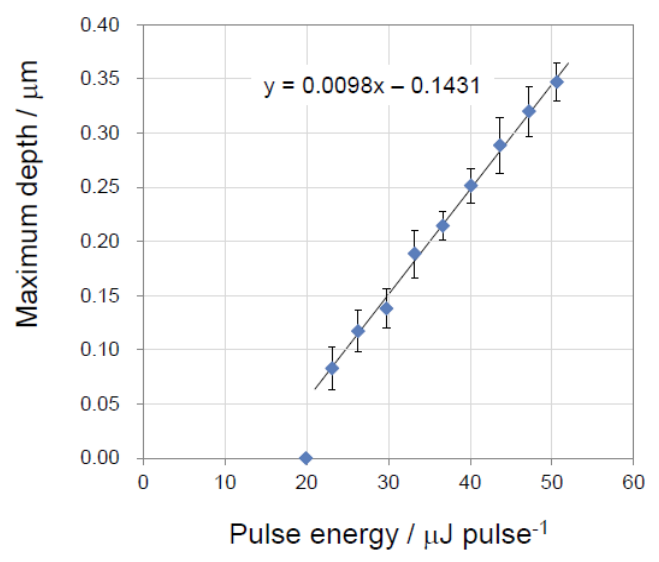

Fig. 4 Maximum depths of micropits obtained by LIBWE with single beam scan (pulse repetition: 10 $\mathrm{kHz}$, scan speed $300 \mathrm{~mm} / \mathrm{s}$ ) at different pulse energy

Depth of micropits also increased by repeating irradiation with scans. Owing to precise pulse delivering by the galvano-scanner, micropit array structures were maintained under repeated scans. Fig. 5 shows maximum depths of micropits fabricated by laser pulses with the energy of 50.6 $\mu \mathrm{J} /$ pulse and various number of scans.

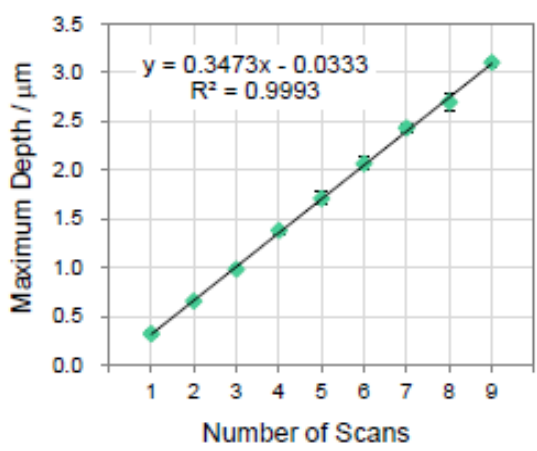

Fig. 5 Maximum depths of micropits fabricated with pulses with the energy of $50.6 \mu \mathrm{J} /$ pulse and various number of scans (pulse repetition: $10 \mathrm{kHz}$, scan speed $300 \mathrm{~mm} / \mathrm{s}$ ).

As the figure shows, maximum depths of micropits increased linearly with number of scans. Etch rate was estimated to be $0.35 \mu \mathrm{m} /$ pulse. This etch rate is much higher than typical etching rates of LIBWE using organic solutions ( $\sim 0 \mathrm{~nm} /$ pulse) [17, 21-23, 32]. Relatively high etch rates (around $100 \mathrm{~nm} /$ pulse) of LIBWE with organic solution were reported [14, 20]: in both cases, etch rate was evaluated from depth of microtrenches fabricated highpulse repetition. When the liquid metallic absorber was employed, much higher etch rates (up to $200 \mathrm{~nm} /$ pulse [24] and $630 \mathrm{~nm} /$ pulse [19]) were shown. Interestingly, higher etch depths up to $200 \mathrm{~nm} /$ pulse by single pulse irradiation were observed in laser-induced dry etching (LIBDE), where thin film absorber was used instead of organic solution [32-34]. Etch rates of LIBDE are reported to show approximately 5-6 times greater than those of LIBWE [32].
Maximum depths of micropits fabricated by single pulse irradiation were $0.347 \pm 0.017 \mu \mathrm{m}$ and $0.320 \pm 0.034$ $\mu \mathrm{m}$ in Figs. 4 and 5, respectively. These values are almost same with the etch rate evaluated from multi scan experiments, indicating that LIBWE with high pulse energies involves practically negligible incubation effect. Such conditions are suitable for fast laser marking of glassware. Similar etching behavior was confirmed and discussed by Tsvetkov et al. [31], recently.

On the other hands, at pulse energy of $19.9 \mu \mathrm{J} /$ pulse, formation of micropits could be observed with multiple scans. Maximum depths of micropits fabricated by laser pulses with the energy of $19.9 \mu \mathrm{J} /$ pulse and various number of scans are shown in Fig. 6. In this case, depths increased linearly with number of scans as well. Etch rate was estimated to be $73 \mathrm{~nm} /$ pulse. The applied pulse energy corresponds to the fluence of $2.8 \mathrm{~J} / \mathrm{cm}^{2}$. At this pulse energy, multiple scan must be employed for laser marking due to incubation effect $[17,21]$.

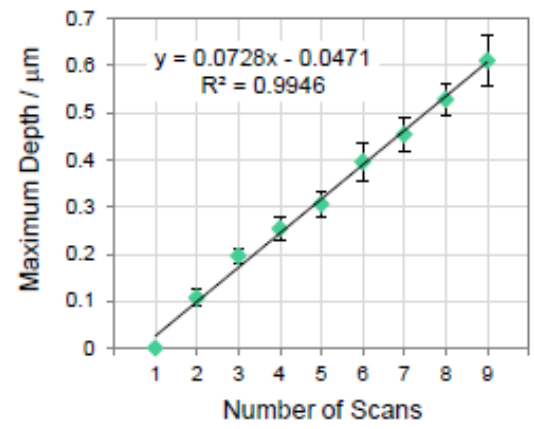

Fig. 6 Maximum depths of micropits fabricated with pulses with the energy of $19.9 \mu \mathrm{J} /$ pulse and various number of scans (pulse repetition: $10 \mathrm{kHz}$, scan speed $300 \mathrm{~mm} / \mathrm{s}$ ).

\subsection{Diagnostic of cavitation}

It is widely known that formation of cavitation bubbles is involved in LIBWE process. As reported before [14], LIBWE under fast pulse repetition results in disturbance of etching due to temporal loss of glass-liquid interface. In the fabrication of micropits, such disturbance could be clearly observed. To clarify how cavitation limit the etching and to set appropriately the maximum repetition rate for fast laser marking, behavior of cavitation was studied in detail.

The formation of cavitation bubbles was studied to evaluate of initial pressure generated in liquid based on expanding velocity of the bubble, which are obtained by transient shadowgraph images [10, 23, 28, 30]. Behavior of cavitation could be also monitored by transient pressure measurements. A pair of signals, which correspond to the formation and collapsing of cavitation bubbles, was detected as reported previously [11, 13, 32]. A typical result obtained by single pulse irradiation with the pulse energy of $44.0 \mu \mathrm{J} /$ pulse is shown in Fig. 7. 


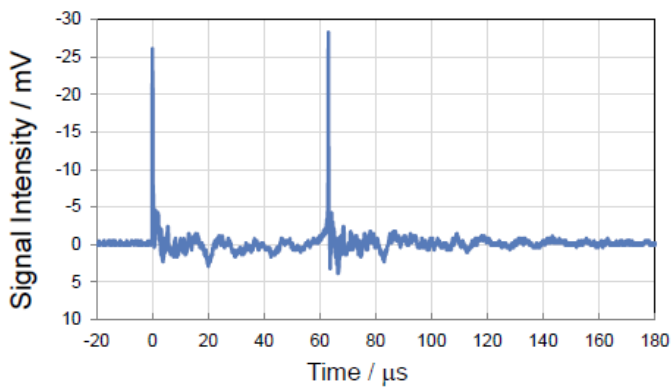

Fig. 7 A pair of pressure signals obtained for single pulse irradiation with energy of $44.0 \mu \mathrm{J} /$ pulse.

The time interval of two signals was $63.2 \pm 0.9 \mu$ s, revealing the lifetime of cavitation bubbles. The lifetime of bubbles increased with pulse energies as shown in Fig. 8.

When the pulses with the energy of $44.0 \mu \mathrm{J} /$ pulse is irradiated at $20 \mathrm{kHz}$ pulse repetition, the lifetime of cavitation bubble (63.2 $\pm 0.9 \mu \mathrm{s})$ is longer than the time interval of laser pulses $(50 \mu \mathrm{s})$. The second pulse is impinged before collapsing the cavitation bubble generated by the first pulse.

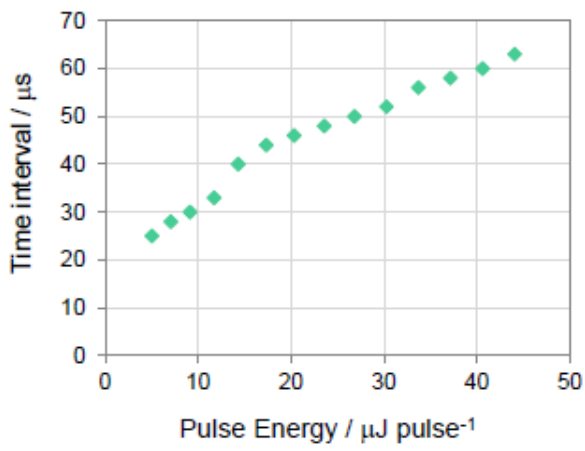

Fig. 8 Time intervals between a pair of pressure signals observed for single pulse irradiation with various energies

Fig. 9 shows micropit arrays fabricated with irradiation at the pulse repetition rates of 10 and $20 \mathrm{kHz}$. Scan speed was set to 750 and $1500 \mathrm{~mm} / \mathrm{s}$, respectively, for delivering the laser pulses to every $75 \mu \mathrm{m}$. Fig. 9(a) show that the micropit array structure with intervals of $74.1 \mu \mathrm{m}$ by irradiation at $10 \mathrm{kHz}$ pulse repetition, revealing that all laser pulses were used for micropit formation. Meanwhile, micropits were fabricated with intervals of $146 \mu \mathrm{m}$ at $20 \mathrm{kHz}$ pulse repetition, nevertheless laser pulses were irradiated at every $75 \mu \mathrm{m}$ (Fig. 9(b)). These results revealed that the second pulse in successive two pulses was not used for micropit formation. This would be caused by temporal lack of glass-liquid interface due to remaining cavitation bubbles. In the present study, actual size of cavitation bubbles could not be evaluated. The maximum radius of the bubble could be related to the lifetime of the bubble as discussed by Zimmer et al. [17]. Moreover, Lee et al. reported that the lifetime of the cavitation bubble with maximum radius of ca. $280 \mu \mathrm{m}$ was ca. $70 \mu$ s [28]. From these results, in the present case, cavitation bubble with maximum radius of ca. $250 \mu \mathrm{m}$ should be generated upon irradiation of laser puls- es with energy of $44.0 \mu \mathrm{J} /$ pulse. To avoid the effect of bubbles, extremely high scan speed must be employed.

To investigate in detail, maximum depths of micropits fabricated by irradiation of two laser pulses with various time intervals were examined. The pulse energy was fixed at $44.0 \mu \mathrm{J} /$ pulse. For each condition, maximum depths of eight micropits were measured and displayed in Fig 10 . The corresponding depths of micropits fabricated by single pulse irradiation was added to the figure at time zero. Their averaged maximum depth was $0.286 \mu \mathrm{m}$.

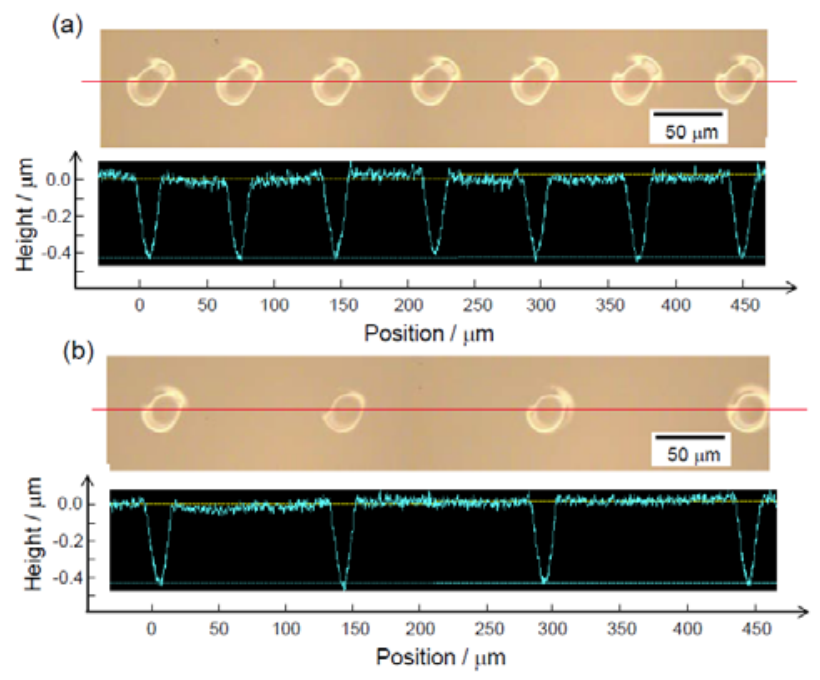

Fig. 9 Micropit array structures (micrographs and surface profiles) fabricated with laser pulses with the energy of $44.0 \mu \mathrm{J} /$ pulse: (a) pulse repetition: $10 \mathrm{kHz}$, scan speed $750 \mathrm{~mm} / \mathrm{s}$ and (b) pulse repetition: 20 $\mathrm{kHz}$, scan speed $1500 \mathrm{~mm} / \mathrm{s}$.

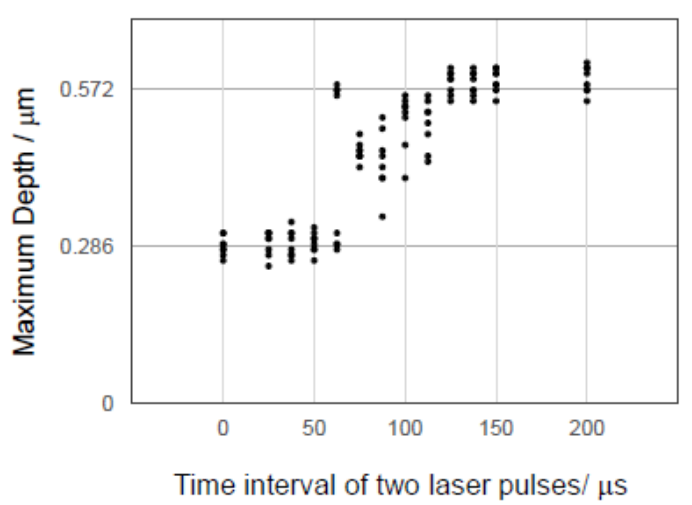

Fig. 10 Maximum depths of micropits fabricated with two laser pulses irradiation with various time intervals and fixed pulse energy of $44.0 \mu \mathrm{J} /$ pulse. Depths of micropits fabricated by single pulse irradiation are added at time zero.

When time interval of two laser pulses was less than 50 $\mu$, depths of fabricated micropits were almost same as those obtained for micropits fabricated by single pulse irradiation. In these cases, the etching by the second pulse was completely suppressed. On the other hand, when time interval of two laser pulses was more than $125 \mu \mathrm{s}$, obtained depths are almost twice of those obtained by single pulse 
irradiation, indicating successful etching by the second laser pulse. These results indicate that existence of glassliquid interface is indispensable for micropit formation by single pulse irradiation.

Interestingly, when time interval was $62.5 \mu \mathrm{s}$, this value is close to the lifetime of cavitation bubbles $(63.2 \pm 0.9 \mu \mathrm{s})$, showed the results divided into two cases: one shows depths for single pulse results and the other shows depths of double pulse results.

When time interval was between 75 and $112.5 \mu$ s, obtained depths were scattered between those obtained by single and double pulses. It was often reported that the collapsing of first cavitation bubble results in formation of second cavitation bubbles [28, 35]. The scattered results would indicate that partial recovering of solid-liquid interface under existence of secondly formed cavitation bubbles resulted in partial etching. Thus, the etching behavior in LIBWE is strongly affected by cavitation. Therefore, setting of pulse repetition rate is important for stable microstructure formation. Micropit array structures fabricated by LIBWE can be used for laser marking of glassware (Fig. 11).

(a)

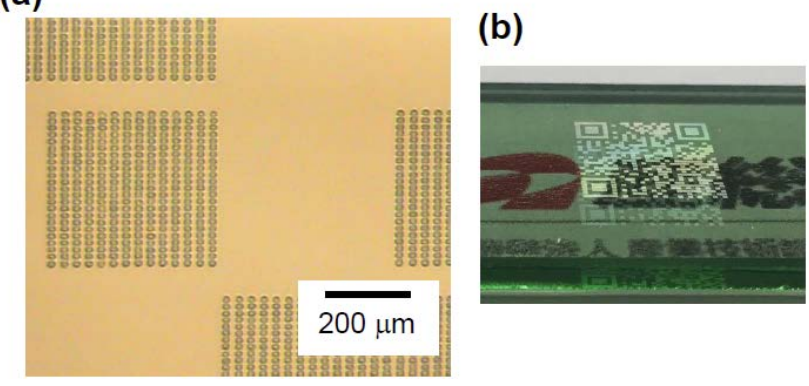

Fig. 11 An example of laser marking of glassware by LIBWE with (a) micropit array structures for writing (b) a QR code on a soda-lime glass plate.

\section{Summary}

For high-speed and flexible laser marking of glassware, fabrication of micropit array structures by LIBWE was investigated. When scan speed is high enough, micropit formation by single pulse irradiation becomes apparent. It was confirmed that micropits with maximum depths up to ca. $0.35 \mu \mathrm{m}$ could be fabricated by single pulse irradiation. Moreover, the depths of micropits could increase by accumulate pulse irradiation without formation of crack.

While formation of micropits was observed upon single pulse irradiation when the applied pulse energy was high enough, at pulse energy of $19.9 \mu \mathrm{J} /$ pulse, formation of micropits could not be observed upon single pulse irradiation due to incubation effect. At the pulse energy, multiple scan is necessary for laser marking.

Under the conditions for fabricating micropits pulse by pulse, interference of etching with cavitation bubbles were clearly revealed. Laser pulse irradiated during temporal loss of glass-liquid interface due to existence of cavitation bubble cannot contribute to etching. Not only firstly generated cavitation bubbles but the secondary generated cavitation bubbles affected the etching. Therefore, setting of pulse repetition rate is important for stable microstructure formation. Micropit array structures fabricated by LIBWE can be used for fast and flexible laser marking of glassware.

\section{Acknowledgments}

This work was supported in part by Regional Innovation Creation R\&D Project (22U30001), Ministry of Economy, Trade and Industry (METI). We thank Mr. K. Kazama, H. Nishizawa, and N. Shimozono of YE Data Inc. for information on QR code reader systems.

\section{References}

[1] Y. M. Noor, S. C. Tam, L. E. N. Lim and A. Jana: J. Mater. Process. Technol., 42, (1994) 95.

[2] J. Qi, K. L. Wang and Y. M. Zhu: J. Mater. Process. Technol., 139, (2003) 273.

[3] X.-S. Li, W.-P. He, L. Lei, J. Wang, G.-F. Guo, T.-Y. Zhang and T. Yue: Opt. Laser Technol., 77, (2016) 31.

[4] V. Veiko, G. Odintsova, E. Ageev, Y. Karlagina, A. Loginov, A. Skuratova and E. Gorbunova: Opt. Exp., 22, (2014) 24342.

[5] B. Dusser, Z. Sagan, H. Soder, N. Faure, J. P. Colombier, M. Jourlin and E. Audouard: Opt. Exp., 18, (2010) 2013.

[6] M. Chen, Y. Chen, W. Hsiao, S. Wu, C. Hu and Z. Gu: Opt. Laser Eng., 46, (2008) 410.

[7] B. Dusser, Z. Sagan, D. Bruneel, M. Jourlin and E. Audouard: J. Phys.:Conf. Ser., 77, (2007) 012002.

[8] T. Dumont, T. Lippert, A. Wokaun and P. Leyvraz: Thin Solid Films, 453-4, (2004) 42.

[9] J. Wang, H. Niino and A. Yabe: Appl. Phys. A, 68, (1999) 111.

[10]H. Niino, Y. Yasui, X. Ding, A. Narazaki, T. Sato, Y. Kawaguchi and A. Yabe: J. Photochem. Photobiol. A: Chem., 158, (2003) 179.

[11]Y. Kawaguchi, X. Ding, A. Narazaki, T. Sato and H. Niino: Appl. Phys. A, 80, (2005) 275.

[12] H. Niino, Y. Kawaguchi, T. Sato, A. Narazaki, T. Gumpenberger and R. Kurosaki: Appl. Surf. Sci., 252, (2006) 4387.

[13] H. Niino, Y. Kawaguchi, T. Sato, A. Narazaki, T. Gumpenberger and R. Kurosaki: J. Laser Micro/Nanoeng., 1, (2006) 39.

[14] T. Sato, Y. Kawaguchi, R. Kurosaki, A. Narazaki, W. Watanabe and H. Niino: J. Laser Micro/Nanoeng., 6, (2011) 204.

[15] T. Nakazumi, T. Sato, A. Narazaki and H. Niino: J. Micromech. Microeng., 26, (2016) 095015.

[16] R. Böhme, A. Braun and K. Zimmer: Appl. Surf. Sci., 196, (2002) 276.

[17] R. Böhme and K. Zimmer: Appl. Surf. Sci., 247, (2005) 256.

[18] R. Böhme, T. Otto and K. Zimmer: Appl. Surf. Sci. 252, (2006) 4392.

[19] K. Zimmer, R. Böhme, D. Ruthe and B. Raushenbach: Appl. Phys. A, 84, (2006) 455.

[20] M. Ehrhardt, G. Raciukaitis, P. Gecys and K. Zimmer: Appl. Surf. Sci., 256, (2010) 7222.

[21] M. Ehrhardt, P. Lorenz, P. Yunxiang, L. Bayer, B. Han and K. Zimmer: Appl. Phys. A,123, (2017) 251. 
[22] G. Kopitkovas, T. Lippert, C. David, A. Wokaun and J. Gobrecht: Microelentron. Eng., 67-8, (2003) 438.

[23] C. Vass, T. Smausz and B. Hopp: J. Phys. D: Appl. Phys., 37, (2004) 2449.

[24] B. Hopp, T. Smausz, C. Vass, G. Szabó, R. Böhme, D. Hirsch and K. Zimmer: Appl. Phys. A, 94, (2009) 899.

[25] J.-Y. Cheng, M.-H. Yen, C.-W. Wei, Y.-C. Chuang and T.-H. Young: J. Micromech. Microeng. 15 (2005) 1147.

[26] Z. Q. Huang, M. H. Hong, K. S. Tiaw and Q. Y. Lin: J. Laser Micro/Nanoeng., 2, (2007) 194.

[27] K. Fujito, T. Hashimoto, K. Samonji, J. S. Speck and S. Nakamura: J. Cryst. Growth, 272, (2004) 370.

[28] T. Lee, D. Jang, D. Ahn and D. Kim: J. Appl. Phys., 107, (2010) 033112.

[29] M. Konstantaki, P. Childs, M. Sozzi and S Pissadakis: Laser Photonics Rev., 7, (2013) 439.
[30] X. Z. Xie, M. F. Hu, W. F. Chen, X. Wei, W. Hu, X. Y. Gao, X. R. Yuan and M. H. Hong: J. Laser Micro/Nanoeng., 8, (2013) 259.

[31] M. Y. Tsvetkov, V. I. Yusupov, N. V. Minaev, A. A. Akovantseva, P. S. Timashev, K. M. Golant, B. N. Chichkov and V. N. Bagratashvili: Opt. Laser Tech., 88, (2017) 17.

[32] B. Hopp, Cs, Vass, T. Smausz and Zs. Bor: J. Phys. D: Appl. Phys., 39, (2006) 4843.

[33] K. Zimmer, M. Ehrhardt, P. Lorenz, X. Wang, C. Vass, T. Csizmadia and B. Hopp: Appl. Surf. Sci., 302, (2014) 42.

[34] Y. Pan, M. Ehrhardt, P. Lorenz, B. Han, B. Hopp, C. Vass, X. Ni and K. Zimmer: Appl. Phys. A 122, (2016) 365.

[35] K. Sasaki, T. Nakano, W. Soliman and N. Takada: Appl. Phys. Exp., 2, (2009) 046501.

(Received: June 7, 2017, Accepted: October 26, 2017) 